\title{
Urinary Tract Aspergillosis in a Patient With Diabetes Who Underwent Total Abdominal Hysterectomy
}

\author{
FARZANA SHARMIN
}

\begin{abstract}
:
Urinary tract aspergillosis is uncommon even in the era of increased frequency of invasive mycoses. Experience is largely from isolated case reports and few case series or reviews. The majority of cases involve transplant recipients predominantly following renal transplantation but is also reported in other immunocompromised states such as AIDS and uncontrolled diabetes mellitus. Most cases represent haematogenous spread to renal parenchyma, often in the absence of recognized focus of infection, presenting as small or large abscesses, infarcts, renal insufficiency or urinary drainage system fungal balls with obstructive uropathy. Diagnosis is usually made on the basis of renal tissue aspiration, urine cultures or by histopathology of the suspicious mass passed through urinary tract. The effective management of invasive aspergillosis includes strategies to optimize prevention and early antifungal treatment, immunomodulation, and, in some cases, surgery. Newer anti-Aspergillus drugs notably voriconazole offer less toxic therapeutic options and are quite successful in combination with drainage measures to relieve urinary stasis. This report describes a case of primary urinary tract aspergillosis with fungal bezoars formation in a 37 years old female, diabetic, hypertensive, who presented with intermittent urinary tract obstruction and was successfully managed by oral voriconazole for six weeks as itraconazole was found refractory.
\end{abstract}

Keywords: Aspergillosis, Urinary tract, Diabetes.

\section{Introduction:}

Fungal infections of urinary tract are well known and symptoms vary from asymptomatic infections to fatal fungaemia. Fungal infections are becoming increasingly common due to overuse of antibiotics, urinary tract instrumentation or loss of immunocompetency in the host ${ }^{1}$. Most of the cases are due to candidal infection, other rare cases include Aspergillus, Cryptococcusetc (1).

Aspergillus species are ubiquitous and exposure to their spores is frequent. Aspergillosis is primarily a pulmonary pathology with haematogenous spread to another organs Primary urinary aspergillosis is rare. Aspergillus species continue to be an important cause of life-threatening infection in immunocompromised patients. This at-risk population is comprised of persons with compromised immune status, such as diabetics, those on corticosteroids and HIV positive individuals or chronic alcoholic patients ${ }^{1,2}$. Patients with prolonged neutropenia, allogeneic hematopoietic stem cell transplant (HSCT), solid organ transplant (SOT), inherited immunodeficiency are also at risk. The most common species is Aspergillus fumigatus, but other species, including $A$. flavus, $A$. terreus, and $A$. niger, also cause disease. The effective management of invasive aspergillosis includes strategies to optimize prevention and early antifungal treatment, immunomodulation, and, in some cases, surgery the recommended management of patients with urinary aspergilloma remains unclear. A case of a lady who presented with aspergillosis of the urinary tract, which was symptomatic and responded to oral antifungal therapy is reported here.

Address of Correspondence: Dr. Farzana Sharmin, Consultant, NHN executive center, Uttara, Dhaka, Bangladesh. Mobile: (+88)01711266708, Email: shila2215@yahoo.com 


\section{Case Report:}

A 37 years old diabetic, hypertensive female, came to outpatient department of the Ibrahim General Hospital, Savar, in August 2016, with history of severe lower abdominal pain with urinary retention which relieved after passage of fleshy mass per urethra one day back. Patient gave history of total abdominal hysterectomy due to fibroid three months back. After one month of operation she experienced severe lower abdominal pain and low-grade fever with burning micturition. The pain was of moderate intensity radiating to both groin, subsided by parenteral analgesics and accompanied by the passage of small, pinkish white soft balls per urethra during the process of urination. The pain subsided significantly following passage of these masses. The episodes of pain and subsequent passage of masses was intermittent, varying from once to twice per week. She had poor compliance with medication of diabetes. General physical examination revealed anaemia, BMl $30 \mathrm{~kg} / \mathrm{m}^{2}$, pulse 86 beats/min, BP 130/ $80 \mathrm{~mm}$ of $\mathrm{Hg}$ with positive suprapubic tenderness. Rest of the systemic examination was markable.

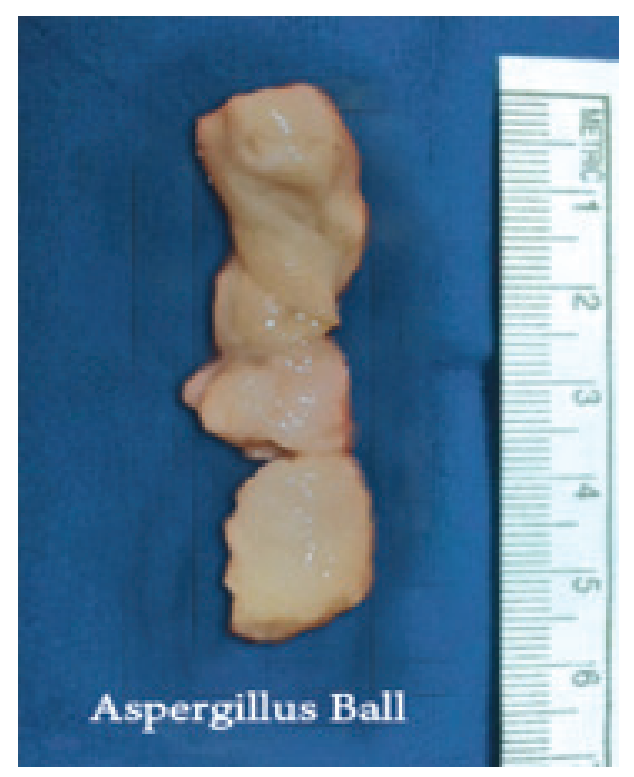

Fig.-1: Fleshy mass of fungus passed per urethra.

The patient underwent hematological investigations to see control of diabetes or any other cause of immunodeficiency. $\mathrm{HbA} 1 \mathrm{C}$ was $8.5 \%$. Her renal and hepatic functions were within normal limits. Blood sample for bacterial cultures was sterile and X-ray chest did not reveal any focus of infection. Ultrasound of whole abdomen shows mild hydronephrosis and fatty liver. Urine R/E shows plenty of pus cells but culture reveals no growth. Histopathological examination of the expelled mass shows aspergillus ball. As the patient refused admission for treatment with amphotericin $\mathrm{B}$, itraconazole was prescribed. Itraconazole $100 \mathrm{mg}$ was given 12 hourly for six weeks. But patient still passed fleshy mass, after three weeks of treatment. So as a better option voriconazole was prescribed for 14 days. Voriconazole $400 \mathrm{mg} 12$ hourly for the first day followed by $200 \mathrm{mg}$ ( 12 hourly) was given. Patient was free of symptoms during treatment time but 7 days after had a recurrence. Again, voriconazole was started for 6 weeks and this time patient remain symptom less for last 6 months. This patient experienced photopsia during early few days and subsided spontaneously.

\section{Discussion:}

Fungal infections of the urinary tract are increasing in incidence, mostly due to the increasing use of antibiotics, instrumentation and indwelling urinary catheters (1).Primary aspergillus infection of the urinary tract is rare (3). Aspergillus species have emerged as an important cause of life-threatening infections in immunocompromised patients. This expanding population is composed of patients with prolonged neutropenia, advanced HIV infection, and inherited immunodeficiency and patients who have undergone allogeneic hematopoietic stem cell transplantation (HSCT) and/or lung transplantation. They may be symptomatic or may remain asymptomatic. There is a predilection for drainage structures rather than the renal parenchyma (2). A spectrum of clinical manifestations varying from asymptomatic colonization (most common), cystitis, pyelonephritis, renal infection, aspergillus balls or bezoars formation, $(3,4)$ ureteric obstruction $(5)$, ureteric colic, renal failure, sepsis (3) or disseminated aspergillosis have been reported(1).Aspergillosis can also involve other genitourinary organs like prostate as reported in literature(5). Aspergillus fumigatus is the most common species recovered from cases of invasive aspergillosis (6). The next most commonly recovered species are Aspergillus flavus, Aspergillus niger, and Aspergillus terreus(7). Except spasmodic pain and discomfort, our patient did not manifest any fever or severe constitutional symptoms. Visible passage of aspergillus balls in urine was frightening 
symptom for the patient. This is rare and has been reported previously in a patient with acute myelocytic leukemia ${ }^{8}$.

It is recommended that tissue and fluid specimens or the expelled mass is to be submitted in adequate quantities for simultaneous histopathologic/cytologic and culture examination. In the case of isolates with atypical growth or concerns for resistance, species identification by molecular methods should be employed.

It is recommended that asymptomatic funguria in hospitalized patients may not be treated as treatment does not impact morbidity and mortality. It may be only a sickness indicator like bacteriuria in elderly ${ }^{9}$. Treatment is recommended only when funguria is symptomatic or in case where host factors increase the risk of fungaemia (1). Reduction of risk factors like removal of indwelling catheters and optimizing antibiotic usage should be tried. In immunocompromised patients, a combined approach with topical and systemic antifungal agents and endo-urological access for extraction, lavage and debulking may be required ${ }^{10}$. However, in immunocompetent, surgical therapy is not necessary. In fact asymptomatic prostatic aspergillosis has been reported to disseminate after a routine transurethral resection procedure ${ }^{11}$.

Aspergillosis is reported to behave more malignantly than candidiasis of urinary tract ${ }^{10}$. Treatment with amphotericin B has previously been recommended (12). Oral itraconazole may be recommended as a first line treatment and has proven effective even in amphotericin B resistant cases ${ }^{10}$. The Infectious Diseases Society of America (IDSA) released updated guidelines for the treatment of aspergillosis in $2016^{13}$. Guidelines recommended voriconazole as initial therapy of invasive aspergillosis. It has been proved to be superior to amphotericin-B in terms of response and fewer side effects ${ }^{11}$. The currently recommended dose of $200 \mathrm{mg}$ orally every 12 hours has been noted to result in low or even unmeasurable serum concentrations in a substantial proportion of patients, and high concentrations may be associated with excessive toxicities ${ }^{14}$. The dose of oral voriconazole can be increased to $4 \mathrm{mg} / \mathrm{kg}$ orally every 12 hours (or $300 \mathrm{mg}$ orally every 12 hours) in patients with disease progression. Voriconazole's profile of adverse reactions includes transient visual disturbances (characterized principally by photopsia); hepatotoxicity, which may be dose limiting (manifested by elevated serum bilirubin, alkaline phosphatase, and hepatic aminotransferase enzyme levels); skin rash (usually in sunlight-exposed areas), visual hallucinations; and others ${ }^{15}$. For patients who are intolerant of voriconazole due to severe reactions, we would choose either a lipid formulation of amphotericin B (eg, AmBisome or Abelcet) or isavuconazole, which was approved by the US Food and Drug Administration (FDA) in 2015. The duration of antifungal therapy is dependent upon the location of the infection, the patient's underlying disease, the need for further immunosuppression, and the response to therapy. Antifungal therapy is generally continued until all signs and symptoms of the infection have resolved and often longer in patients with persistent immune defects. Radiographic abnormalities should have stabilized and signs of active infection should have disappeared before treatment is discontinued. The duration of therapy is 6 to 12 weeks $^{13}$ but, for most immunosuppressed patients, antifungal therapy will continue for months or even years in some cases.

\section{Conclusion:}

The present case emphasizes the possibility of iatrogenic urinary fungal infection secondary to instrumentation in diabetic person. A high index of suspicion in such patients and careful investigations are needed to establish the diagnosis. Moreover, prompt treatment with recommended drug is required to reduce the morbidity of the patient.

\section{References:}

1. Haq JA, Khan MA, Afroze N, Haq T. Localized primary renal aspergillosis in a diabetic patient following lithotripsy-a case report. BMC infectious diseases. 2007;7(1):58.

2. Smaldone MC, Cannon Jr GM, Benoit RM. Case report: bilateral ureteral obstruction secondary to Aspergillus bezoar. Journal of endourology. 2006;20(5):318-20.

3. Shohab D, Hussain I, Khawaja A, Jamil I, Raja $\mathrm{NU}$, Ahmed F, et al. Primary renal aspergillosis and xanthogranulomatous pyelonephritis in an immuno-competent toddler. J Coll Physicians Surg Pak. 2014;24(Suppl 2):S101-3.

4. Ahuja A, Aulakh BS, Cheena DK, Garg R, Singla $S$, Budhiraja S. Aspergillus fungal balls causing 
ureteral obstruction. Urology journal. 2009;6(2):127-9.

5. Abbas F, Kamal MK, Talati J. Prostatic aspergillosis. The Journal of urology. 1995;153(3 Pt 1):748-50.

6. Perfect J, Cox G, Lee J, Kauffman C, De Repentigny L, Chapman S, et al. The impact of culture isolation of Aspergillus species: a hospital-based survey of aspergillosis. Clinical Infectious Diseases. 2001;33(11):1824-33.

7. Walsh T, Groll A. Overview: non-fumigatus species of Aspergillus: perspectives on emerging pathogens in immunocompromised hosts. Current opinion in investigational drugs (London, England: 2000). 2001;2(10):1366.

8. Guleria S, Seth A, Dinda AK, Kumar R, Chabbra $\mathrm{R}$, Agarwal SK, et al. Ureteric aspergilloma as the cause of ureteric obstruction in a renal transplant recipient. Nephrology, dialysis, transplantation: official publication of the European Dialysis and Transplant AssociationEuropean Renal Association. 1998;13(3): 792-3.

9. Hartman BJ, Coleman M, Brause BD, Saletan $\mathrm{S}$. Localized renal aspergillosis with hairy cell leukemia: a review of urinary tract aspergillosis in malignant and nonmalignant conditions. Cancer investigation. 1984;2(3):199-202.
10. Kueter JC, MacDiarmid SA, Redman JF. Anuria due to bilateral ureteral obstruction by Aspergillus flavus in an adult male. Urology. 2002;59(4):601.

11. Herbrecht R, Denning DW, Patterson TF, Bennett JE, Greene RE, Oestmann J-W, et al. Voriconazole versus amphotericin B for primary therapy of invasive aspergillosis. New England Journal of Medicine. 2002;347(6):408-15.

12. Vuruskan H, Ersoy A, Girgin N, Ozturk M, Filiz G, Yavascaoglu I, et al., editors. An unusual cause of ureteral obstruction in a renal transplant recipient: ureteric aspergilloma. Transplantation proceedings; 2005: Elsevier.

13. Patterson TF, Thompson III GR, Denning DW, Fishman JA, Hadley S, Herbrecht R, et al. Practice guidelines for the diagnosis and management of aspergillosis: 2016 update by the Infectious Diseases Society of America. Clinical Infectious Diseases. 2016;63(4):e1-e60.

14. Marr KA, Carter RA, Boeckh M, Martin P, Corey $\mathrm{L}$. Invasive aspergillosis in allogeneic stem cell transplant recipients: changes in epidemiology and risk factors. Blood. 2002;100(13):4358-66.

15. Boucher HW, Groll AH, Chiou CC, Walsh TJ. Newer systemic antifungal agents. Drugs. 2004;64(18):1997-2020. 